EOMmun Communication et organisation

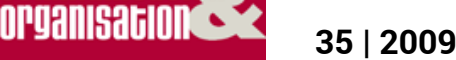

Repenser la communication dans les organisations publiques

\title{
Repenser la communication des organisations publiques
}

Elizabeth Gardère et Amar Lakel

\section{OpenEdition}

\section{Journals}

Édition électronique

URL : http://journals.openedition.org/communicationorganisation/685

DOI : 10.4000/communicationorganisation.685

ISSN : $1775-3546$

Éditeur

Presses universitaires de Bordeaux

Édition imprimée

Date de publication : 1 juin 2009

Pagination : 7-12

ISSN : 1168-5549

Référence électronique

Elizabeth Gardère et Amar Lakel, «Repenser la communication des organisations publiques », Communication et organisation [En ligne], 35 | 2009, mis en ligne le 01 décembre 2009, consulté le 10 décembre 2020. URL : http://journals.openedition.org/communicationorganisation/685 ; DOI : https:// doi.org/10.4000/communicationorganisation.685 


\title{
Repenser la communication des organisations publiques
}

\author{
Elizabeth Gardère, Amar Lakel \\ elizabeth.gardere@u-bordeaux4.fr \\ amar.lakel@u-bordeaux3.fr
}

Les gouvernements, administrations, institutions, collectivités et de manière plus générale, les organisations publiques s'appuient de manière croissante sur des processus d'action collective dans un contexte de crise de légitimité de la représentation politique en France. Les enjeux communicationnels sont prégnants. De la communication politique, publique, institutionnelle en passant par la communication locale, promotionnelle, voire marketing, l'étendue du champ lexical et des pratiques est vaste et trop d'amalgames sont encore au cœur de controverses.

Aussi, la pérennisation de la communication institutionnelle publique questionne-t-elle la définition de cette même activité organisationnelle née dans un flou conceptuel en s'inspirant des modèles d'usage entrepreneuriaux, voire électoraux. Tandis que, dans la pratique, elle est connue et largement mise en oeuvre, le concept tarde à être théorisé. Les postures varient entre les disciplines et les auteurs. De l'association des termes à la distinction nette entre la communication politique et la communication publique, le concept n'est pas encore stabilisé. Pourtant un glissement de paradigme est déjà engagé et concerne l'impact organisationnel où la communication est intégrée par les acteurs comme élément de gestion des services publics (Bessières). Par ailleurs, le débat reste ouvert sur la représentation de l'institution communicante qui relève d'un artefact ou bien qui constitue un modèle valide pour analyser la communication des organisations publiques (Bonnet, Hélaine-Pinsard).

Mentionner la communication locale, c'est aussi considérer que toute personne a le droit de prendre part à la direction des affaires publiques de son pays, de sa ville, de son administration, soit directement, soit par l'intermédiaire de représentants librement choisis. Dans la pratique, la représentation par mandat électif peut être doublée d'une concertation directe. En effet, si la communication locale donne aux citoyens les moyens de juger les actions et choix publics en faisant connaître les objectifs politiques des élus, a contrario, une communication définie a posteriori ne favorise pas l'intégration sociale dans l'espace local. Cela implique une réflexion a priori qui 
contribuera à l'organisation sociale de l'espace collectif. Toutefois, parler de démocratie participative, c'est remettre en cause le processus de démocratie représentative. La temporalité démocratique est alors reconsidérée. Elle n'est plus dédiée aux périodes électorales, mais s'inscrit dans des logiques de projet à moyen et long terme. (Gardère, 2008).

Joseph Schumpeter mettait déjà en exergue dans son ouvrage Capitalisme, socialisme et démocratie une relation inversée: si l'élection est l'expression de la démocratie, alors le pouvoir n'est plus délégué, il est confié : «Il ne s'agit plus d'un gouvernement du peuple, mais d'un gouvernement approuvé par le peuple». Face aux mutations de la communication publique, l'utilisation du dispositif de débat public participatif tend à montrer que l'enjeu n'est pas tant de faire connaître l'action publique à travers la communication territoriale mais davantage de faire admettre l'action publique en ayant recours à la communication (Romeyer). Constat qui s'explique, entre autres, par une situation de crise où la visibilité de l'action publique est l'un des critères pour en assurer l'efficacité et l'efficience. Une stratégie d'alliance entre les médias et les acteurs politiques ouvre alors la voie à de nouvelles logiques d'action publique. L'information médiatique prend le relais des institutions normatives dont le rôle était davantage de cadrer les débats publics (Fallon, Joris).

L'ensemble de ces pratiques interroge le rapport entre la communication et la participation. L'analyse repose sur les images et mots véhiculés par le concept de participation et sur leurs représentations dans le discours de l'homme politique local. L'hypothèse repose sur l'intérêt croissant accordé par les autorités publiques à l'implication des citoyens dans l'évaluation des politiques publiques. Intérêt qui tend à pervertir le sens de la participation (Monseigne). Mais dès lors que la communication est assimilable à de l'information, la pratique de la participation est réduite à sa plus simple expression (Huron, Spieth).

L'importance accordée à la gestion de l'information remonte au XIX siècle. Préalablement à la consultation directe au niveau local, la loi du 18 juillet 1837 instaurait l'accès public aux documents administratifs. Toutefois, cette communication avait ses limites. Elle était réservée aux contribuables de la collectivité. Il faut attendre que les conseils de l'assemblée délibérante deviennent publics, et que les décisions ainsi que les motivations de ces décisions soient portées à la connaissance du grand public pour élargir l'accès à l'information. Cette pratique permet un meilleur contrôle des administrateurs et une 
meilleure compréhension de leur action. La motivation de décision est obligatoire dans certains cas fixés par le législateur. Elle vise à informer le citoyen de l'impact des opérations engagées et tend à coordonner les interventions publiques. L'information circule de deux façons : d'une part grâce à la mise à disposition des informations transmises par les collectivités ; d'autre part avec les commissions de concertation publique. Informer les citoyens est une pratique démocratique qui suppose le dialogue entre deux types d'acteurs : le citoyen et son représentant (politique ou administratif). Cependant, comme pour l'information, les conditions de mise en place d'une consultation sont restrictives. En effet, réunir des administrés pour leur présenter un projet implique la prise en compte et la mise en valeur de leur opinion. Bien que le cadre de négociation entre les parties soit fixé et parfois restreint, le citoyen peut faire partager son expérience personnelle et ses attentes. Si la consultation est un espace où s'exerce la liberté d'expression sur la chose publique, elle reste avant tout une démarche d'écoute des administrés. En effet, on relèvera des limites à la consultation comme l'état du projet souvent trop avancé au moment de la consultation et qui ne laisse qu'une part infime aux suggestions et la confrontation entre les intérêts collectifs et privés qui reste un point sensible (Gardère 2008).

Bien que les dernières lois introduisent la notion de communication de proximité, ce mode d'action démocratique n'est pas encore devenu une pratique habituelle pour les élus dans le pilotage d'un projet. Elle demande à chacun un changement d'état d'esprit qui conduit à prendre en compte non seulement des critères d'évaluation tels que le respect des délais, la consommation budgétaire et l'atteinte des objectifs, mais aussi le paramètre électoral. La bonne marche d'un projet de démocratie locale représentative et participative implique la maîtrise des outils de communication politique pour déterminer les informations auxquelles les administrés ont accès. Les écueils les plus fréquents dans cet exercice sont l'incohérence du niveau d'information entre les parties en présence, ainsi que les digressions qui amplifient l'incompréhension dans les débats.

$\mathrm{Si}$ certaines pratiques sont consultatives, participatives ou représentatives, d'autres restent plus traditionnelles. Afin d'accroître le niveau d'information délivré aux riverains, divers modes de communication sont utilisés. En plus d'exiger une forte mobilisation, une information accessible et un intérêt accru des médias pour les questions locales, l'exercice de la démocratie locale exige des ressources et des moyens. Le Web 2.0 en est l'illustration la plus avancée (Zémor). L'information des citoyens n'est pas seulement une 
condition du bon fonctionnement de la démocratie locale, elle est aussi nécessaire pour faciliter une bonne administration locale. À cette condition, l'information est garante de la gestion transparente de la collectivité. Toutefois, la transparence et la réciprocité intégrale dans l'espace sociopolitique peuvent-elles être réelles ? Certains dénoncent l'illusion de transparence et d'accessibilité des outils de communication et l'appropriation du pouvoir local sous-entendus par les opérations de concertation (Paillart, 1993). Un administré impliqué dans la gestion de sa collectivité et qui dispose des éléments qui concourent aux prises de décisions politiques, est-il plus à même de prendre part activement à une concertation ?

Les conseils de quartier apparaissent comme l'une des institutions clefs d'un pouvoir consultatif attestant de l'exigence démocratique et de ses difficultés. Malgré de nombreuses expériences, l'enquête réalisée à Strasbourg, atteste de «l'absence de compétence citoyenne » comme « une objection qui apparaît fréquemment dans les mises en œuvre du pouvoir consultatif, et notamment dans les conseils de quartier ». Si des résistances à la montée du pouvoir consultatif persistent et dressent un bilan négatif de leur mise en place, c'est principalement car ces instances ne mettent pas en œuvre le principe de séparation des pouvoirs (Breton, Gissinger).

À travers le cas du territoire rennais, le jeu des acteurs politiques et administratifs affiche un double objectif tant en termes de communication publique locale que de marketing territorial. Ce constat atteste d'un décalage entre les discours et les pratiques des acteurs publics institutionnellement liés. Cela, qu'il s'agisse de services, ou du jeu de pouvoir entre la communication locale, extralocale, voire des enjeux liés à la concurrence managériale entre deux échelles territoriales qui communiquent sur un même espace dans la perspective de la métropolité (Houllier-Guibert). La Communauté d'Agglomération de Nice Côte d'Azur offre un autre terrain pour analyser les objectifs stratégiques d'un établissement public de coopération intercommunale et la communication mise en place. Une large place est laissée aux citoyens en termes d'approches participatives dans les actions de communication intercommunale ce qui permet d'envisager la communication intercommunale entre un agir communicationnel et une gouvernance démocratique (Huron, Spieth). Enfin, face au déficit de légitimité populaire de l'Union européenne, une analyse de la politique de communication impliquera la responsabilité de chaque État membre d'appliquer la politique de communication déterminée collectivement. Internet étant ici proposé comme un levier d'information des citoyens, de promotion et de 
légitimation (Le Saëc). Pour sa part, le Ministère de la Justice ayant fait l'objet d'une déconcentration de la communication avec des dispositifs spécifiques, est soumis à de changements opérationnels liés à l'arrivée de nouveaux acteurs - les magistrats - dans le processus de communication, et notamment dans leurs relations avec les médias. Le constat est qu'au-delà de l'institutionnalisation de la communication, le Ministère opère avec une communication «par le bas » avec de nouveaux acteurs et des critères d'efficacité spécifiques (Thomas). Question qui d'un point de vue interculturel, trouve son corollaire dans l'émergence de la communication publique dans le monde arabe d'après une approche comparative qui questionne les rapports à l'Etat, à la société et à la culture selon les dynamiques liées à l'inscription dans différents contextes culturels (Hammami).

Par ailleurs, l'exemple de la communication événementielle est révélateur des pratiques de communication institutionnelle et de leurs conséquences. Le cas d'une communication culturelle décentralisée montre que dans un contexte de concurrence des territoires, les pratiques publiques, notamment ici dans les projets culturels de type événementiel, sont corrélées aux enjeux sociétaux et aux discours identitaires du territoire. Cela met en évidence la complexité des interactions entre les acteurs dans le processus de médiation où sont imbriqués des phénomènes à la fois politique, culturel et communicationnel (Appel, Boulanger). Exemple complété par la mise en œuvre de la PAC qui, plutôt que d'être une campagne de communication directe, a contribué à la réorganisation de l'institution pour permettre l'encadrement des processus d'agencements entre les acteurs (agriculteurs, scientifiques, industriels...). L'enquête menée porte sur l'influence de cet événement pour mettre en exergue les enjeux d'une nouvelle politique agricole et encourager les acteurs à se tourner vers des pratiques d'agriculture durable. Une double étude compréhensive et analytique des entretiens menés à Agro-Transfert concourt au résultat suivant : la création de réseaux d'actions d'acteurs hétérogènes est une "force canalisatrice » pour mener à bien une politique, la PAC, malgré des instabilités inhérentes au projet comme les interprétations contradictoires. L'interaction est ici le lieu d'intégration progressive de l'action en complément d'une procédure de légitimation (Gkouskou-Giannakou, Huet, Choplin, Lenay).

De même, l'Université s'inscrit dans des logiques d'autonomie et de système concurrentiel qui engagent sa visibilité relayée par des discours politiques, institutionnels et médiatiques qui attestent d'un nouveau langage et d'une démarche de benchmarking. Le langage de la performance, le classement, l'évaluation et les pratiques de 
communication sont devenus les incontournables de l'institution universitaire (Granget).

Face à la demande citoyenne et à l'obsolescence des modèles en vigueur, divers dispositifs ont été mis en œuvre avec des objectifs de participation et de concertation des citoyens avec davantage de communication institutionnelle publique. Aussi, la décision territoriale s'accompagne-t-elle désormais du modèle participatif qui demande toutefois une analyse pour déterminer le moyen et les outils les plus adéquats en fonction de facteurs endogènes, exogènes et de la volonté politique. Cela d'autant que le contexte socio-économique est déterminant et critique. Il faut aussi compter avec les paramètres financiers de la collectivité et le côté procédural qui concerne la délibération nécessaire à l'exercice constructif de la démocratie participative.

Qu'il s'agisse du modèle managérial basé sur une forte coopération avec les acteurs économiques, du modèle de la modernisation participative qui concerne le fonctionnement administratif du service public où la participation s'applique aux usagers dans une relation topdown et où la responsabilité des participants est secondaire; du modèle de démocratie de proximité qui tend vers le maintien de la paix sociale et s'exerce en favorisant le dialogue entre les représentants et les représentés; du modèle de l'empowerment caractérisé par un Etat faible et une fonction prédominante des associations de type $\mathrm{ONG}$; ou du modèle de démocratie participative (Gardère, 2008) qui offre la possibilité à l'Etat de conserver un pouvoir incontestable tout en prônant l'autonomie et la participation de la société civile. Les flux top-down et bottom-up interagissant dans le but de trouver un consensus où les intérêts des parties prenantes sont représentés, la communication institutionnelle publique est ici soumise à l'analyse théorique et au retour d'expérience qui en dressent un bilan conceptuel, empirique et pratique.

Elizabeth Gardère 